\title{
Detecting single and multiple atmospheric secondaries in an 18NM64
}

\author{
Alejandro Sáiz*, Warit Mitthumsiri, David Ruffolo \\ Department of Physics, Faculty of Science, Mahidol University, Bangkok 10400, Thailand \\ E-mail: alejandro.sai@mahidol.ac.th, warit.mit@mahidol.ac.th, \\ david.ruf@mahidol.ac.th
}

Paul Evenson

Bartol Research Institute, University of Delaware, Newark, DE 19716, USA

E-mail: evenson@udel.edu

\section{Tanin Nutaro}

Department of Physics, Faculty of Science, Ubon Ratchathani University,

Ubon Ratchathani 34190, Thailand

E-mail: tnutaro@yahoo.com

\begin{abstract}
We present an update on the analysis of cross-counter time-delay histograms with $5 \mu$ sins as recorded since late 2015 in the Princess Sirindhorn Neutron Monitor, an 18NM64 (with 18 counter tubes in a continuous row) at $2560 \mathrm{~m}$ altitude in Doi Inthanon, Thailand. A cross-counter relative leader fraction $L_{i j}$ can be defined as the fraction of pulses measured in counter number $i$ that are uncorrelated in time with the most recent pulse in counter $j$. For low tube separation, $L_{i j}$ rapidly increases with increasing tube separation $|i-j|$, as expected from the spatial spreading of neutrons produced by the same atmospheric secondary particle inside the neutron monitor. At high tube separation, $L_{i j}$ increases slowly with increasing tube separation and remains different from unity, which we interpret as due to multiple atmospheric secondaries arriving in rapid succession after originating from the same primary cosmic ray. We study temporal variations of the cross-counter relative leader fraction at large tube separation $(|i-j|>6$, or roughly more than $3 \mathrm{~m})$ and analyze the possible contribution of atmospheric effects to the data.
\end{abstract}

36th International Cosmic Ray Conference -ICRC2019-

July 24th - August 1st, 2019

Madison, WI, U.S.A.

\footnotetext{
*Speaker.
} 


\section{Introduction}

Neutron monitors (NMs) are ground-based detectors of 'secondary' cosmic rays (mostly neutrons) that are produced in Earth's atmosphere by interactions of ('primary') cosmic rays with energies $\sim 1 \mathrm{GeV}$ and above. In an NM, a number of cylindrical, gas-filled proportional counters operate inside several tons of $\mathrm{Pb}$ (the producer) to detect individual 'tertiary' neutrons produced from the collisions of atmospheric secondary cosmic rays with $\mathrm{Pb}$ nuclei. The rate of detection, and its variations, can then be related to the flux of primary cosmic rays arriving to Earth.

The Princess Sirindhorn Neutron Monitor (PSNM) is located on the top of Doi Inthanon, Thailand's highest mountain, at latitude $18.59^{\circ} \mathrm{N}$, longitude $98.49^{\circ} \mathrm{E}$, and $2560 \mathrm{~m}$ above sea level. The geomagnetic cosmic ray cutoff there is higher than at any other NM location around the world. The main detector contains 18 neutron counters in a continuous row following the NM64 design [1]; 3 other counters operate outside the $\mathrm{Pb}$ producer (bare counters).

As explained previously [2], the data acquisition system at the PSNM records also the absolute time of each neutron detection, with a few $\mu$ s accuracy. The goal of this extra, non-standard measurement is to have an estimation of cosmic ray energies as well as flux. The distributions of time delays, or times between consecutive neutron detections, can be analyzed to extract a leader fraction $L$ that quantifies the number of detections that are statistically uncorrelated with the previous detection, relative to the total [3]. Typically, two neutron detections that originate from two different cosmic rays are uncorrelated in time as in a Poisson process; on the other hand, tertiary neutrons produced by the same atmospheric secondary in the $\mathrm{Pb}$ are detected within a few $\mathrm{ms}$ and are statistically correlated. Thus $L$ can be shown to be related to the energy spectrum of cosmic rays, since production in the $\mathrm{Pb}$ increases for higher cosmic ray energy.

In our previous work $[3,4,5,6]$, time delay distributions were studied for consecutive detections in the same neutron counter, and $L$ was calculated for each of the 18 counters independently. As noted above, this measurement is dominated by neutrons produced together in the $\mathrm{Pb}$ (from the same secondary), which most of the times are detected in the same counter but often get detected by different counters. Also, some time correlation may arise from the detection of different atmospheric secondaries that were produced together in the atmosphere (i.e., from the same primary). For the sake of understanding these processes, in late 2015 the electronics in the PSNM acquisition system were upgraded to allow for cross-counter time delay analysis. In this work, we present an update on the study of time correlations between neutrons detected in different counters, using cross-counter leader fractions, and analyze the effects of atmospheric pressure and humidity in our measurements, with the aim of studying time variations that could be associated to changes in cosmic ray spectrum.

\section{Definition of cross-counter leader fractions}

As in our previous report [2], we define a cross-counter time delay $t$ from counter $i$ to counter $j$, for each detection in counter $i$, as the time until the earliest detection in counter $j$. The distribution of time delays typically shows an excess of events at short times that arises from correlated neutron detections, on top of an exponential distribution that corresponds to the chance coincidence of uncorrelated secondary neutrons (produced by different primary cosmic rays). For a normalized 
distribution of time delays $n_{i j}$, said exponential tail can be expressed as

$$
n_{i j}(t) \rightarrow \alpha_{j} L_{i j} \exp \left(-\alpha_{j} t\right) \quad t>t_{\text {trans }}
$$

for a transition time $t_{\text {trans }}$ of a few ms, where $\alpha_{j}$ is the probability per unit time of a chance coincidence in counter $j$, and the cross-counter leader fraction $L_{i j}$ represents the probability that a count on counter $i$ is not temporally associated with any later count on counter $j$ [2].

To reduce the number of recorded distributions and increase the statistics, we initially analyzed the 18 combined distributions of cross-counter time delays at a given counter separation $\Delta$, or $n_{\Delta}(t)$, with $\Delta=|i-j|$, for $\Delta=0, \ldots, 17$. There is, however, an intrinsic difference in the values of the chance coincidence probability $\alpha_{j}$ from counter to counter that suggests that these combined distributions $n_{\Delta}(t)$ may deviate from the simple exponential form described above. The most important discrepancy in the values of $\alpha_{j}$ appears between counters at the 'end' positions of the continuous row of counters (first and last counter) and the other 'middle' counters. The value of $\alpha_{j}$ is typically $\sim 15 \%$ lower at end counters, mainly due to the smaller amount of $\mathrm{Pb}$ surrounding these counters. Thus, since August, 2017, the number of recorded distributions was increased to the 50 different combinations for which the counters detecting the early and late neutrons were either a 'middle' (m) or 'end' (e) counter: $n_{\Delta}^{\mathrm{mm}}(t), n_{\Delta}^{\mathrm{me}}(t), n_{\Delta}^{\mathrm{em}}(t)$, and $n_{\Delta}^{\mathrm{ee}}(t)$.

The corresponding 50 values of cross-counter leader fraction can be labelled $L_{\Delta}^{\mathrm{mm}}$ with $\Delta=$ $0, \ldots, 15, L_{\Delta}^{\mathrm{me}}$ and $L_{\Delta}^{\mathrm{em}}$ with $\Delta=1, \ldots, 16$, and $L_{\Delta}^{\mathrm{ee}}$ with $\Delta=0$ and 17 . The two cases with $\Delta=0$ (zero separation) are similar to the same-counter leader fractions studied in our previous work, but combined for the 16 middle counters or the 2 end counters. As in previous work, a fit of each time delay distribution to an exponential function for a specific range of $t$ (50-200 $\mathrm{ms}$ in this case) is used to estimate the corresponding cross-counter leader fraction after proper normalization.

\section{Results for accumulated distributions}

Total distributions of cross-counter time delays were accumulated between August 5th, 2017 and August 31st, 2018 for a total of $\sim 9000 \mathrm{~h}$. Figure 1 shows the cross-counter leader fraction $L_{\Delta}$ as a function of counter separation $\Delta$ for that period, for the different 'middle'- 'end' combinations. The values start at less than 0.8 for separation zero (same counter) and quickly increase when considering cross-counter time delays between distant counters, approaching a constant value close to 1 . A value of $L_{\Delta}=1$ would correspond to totally uncorrelated neutron detections between counters (a Poisson process). However, the value of $L_{\Delta}$ that is observed, which seems to vary little for separations 6-17, is never consistent with 1 .

Among the four 'middle'- 'end' combinations, values of $L_{\Delta}$ at small counter separation $(\Delta<3)$ are higher when the counter on which the early neutron was detected is of the 'middle' type than when it is an 'end' type. Assuming these $L_{\Delta}$ are related to groups of same-secondary events, the mechanism to explain this can be the same that is at play in the same-counter $L$ reported previously [3], for which values of $L$ are lowest at the two end counters and even slightly lower at the second-from-end positions than for middle counters. The smaller amount of $\mathrm{Pb}$ surrounding end counters may account for a smaller fraction of 'leaders', or the proximity of the lateral polyethylene reflector slabs reflecting tertiary neutrons back may generate an increase in the number of 'followers', or both. 

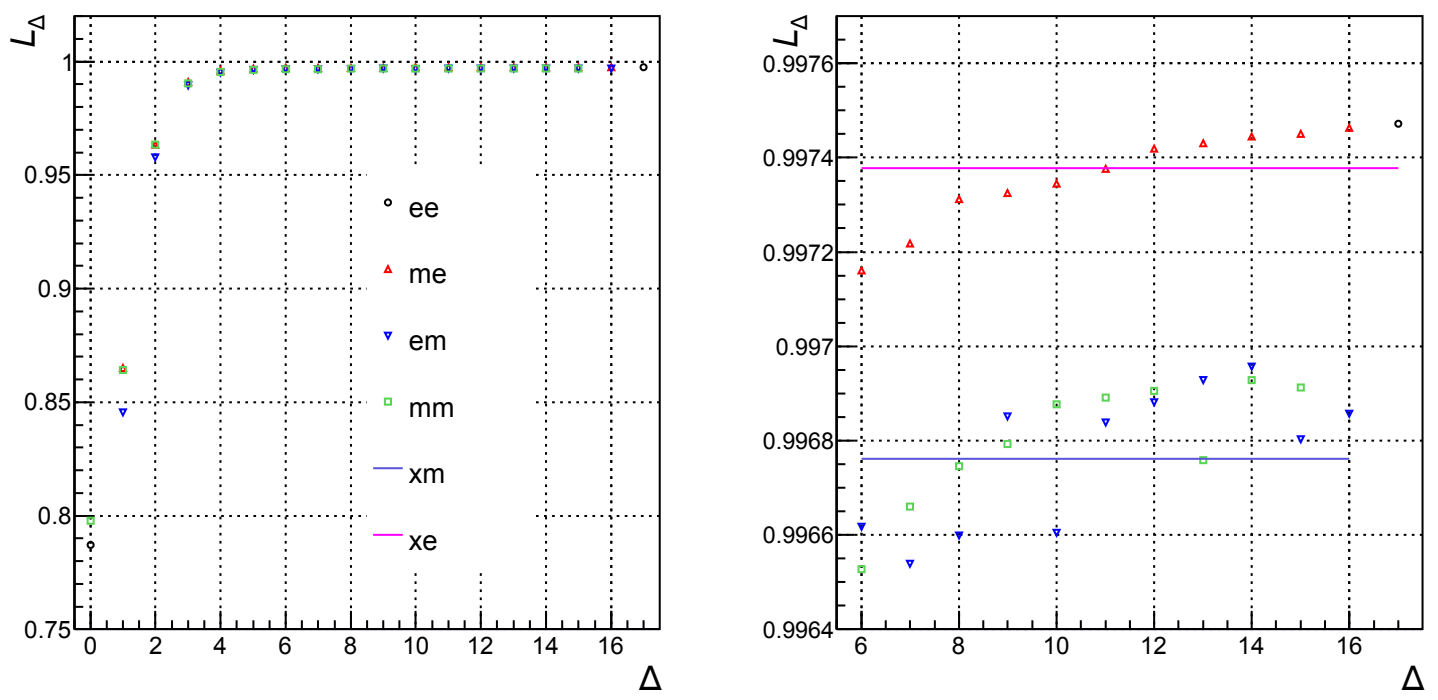

Figure 1: Cross-counter leader fraction $L_{\Delta}$ as a function of counter separation $\Delta$.

On the other hand, the large separation values of $L_{\Delta}$ are mostly determined by the type of counter on which the late neutron is detected. Based on this, we also analyze combined distributions for all separations of 6 positions or more for cases ' $\mathrm{mm}$ '+ 'em' and 'me'+'ee', giving two values of large-separation leader fraction $L_{\Delta>5}^{\mathrm{xm}}$ and $L_{\Delta>5}^{\mathrm{xe}}$, respectively, shown as horizontal lines on the right panel of Figure 1. Both values are consistent with the $L_{\Delta>5}$ value of 0.997 reported previously for an earlier time period and all counter combinations with separation 6 or larger [2].

For these large separation cases, $L_{\Delta}$ is related to multiple secondaries and its higher value when the later counter is of 'end' type is related to these counters being less efficient at registering counts (by $\sim 15 \%$, see above), which makes the multiplicity lower in this case compared to the case when the later counter is of 'middle' type (note that the average multiplicity is $1 / L$ ). These two values $\left(L_{\Delta>5}^{\mathrm{xm}}\right.$ and $\left.L_{\Delta>5}^{\mathrm{xe}}\right)$ are very similar but also close to 1 , so the corresponding 'follower fractions' $1-L_{\Delta}$ actually differ by about $20 \%$ between the two cases.

\section{Results for hourly distributions}

Values of the different cross-counter leader fractions were also calculated for each hour in the period between August 5th, 2017 and August 31st, 2018. As is usual with the same-counter leader fraction [3], changes in atmospheric pressure produce a very small change in the measured value of $L$, but variations in atmospheric water vapor (quite pronounced between the rainy and dry seasons) have a larger effect. Figure 2 shows three examples of correlations between hourly values of $L_{\Delta}$ and the atmospheric pressure and humidity. Variations with pressure (top panels) are more easily identified by subtracting the daily average on both $P$ and $\log L_{\Delta}$. The correlation (fit line) can be used to remove pressure effects. Subsequently, a correlation between pressure-corrected $L_{\Delta}$ and humidity can be identified and a further correction applied to $L_{\Delta}$. As explained elsewhere [6], we characterize atmospheric humidity using the precipitable water vapor (PWV) determined from the GDAS (Global Atmospheric Data Assimilation) database (see http://ready.arl.noaa.gov/gdas1.php). 

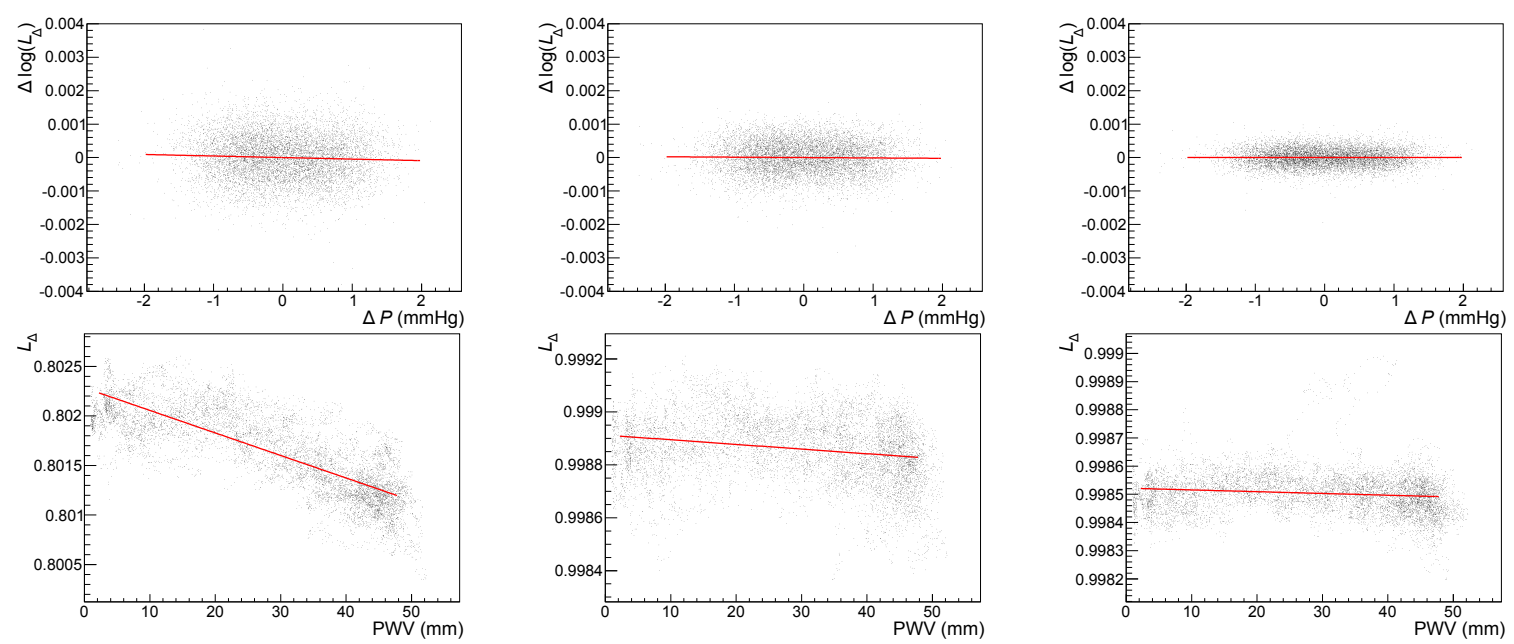

Figure 2: Dependence of $L_{\Delta}$ on (top panels) atmospheric pressure and (bottom panels) atmospheric humidity for the cases (left to right) $L_{\Delta=0}^{\mathrm{mm}}, L_{\Delta>5}^{\mathrm{xe}}$, and $L_{\Delta>5}^{\mathrm{xm}}$.

In Figure 2, one of the same-counter leader fractions is presented, for comparison purposes, together with the two combined large-separation leader fractions. For the latter, the effects of atmospheric humidity appear to be less important; however, considering the follower fraction $1-L_{\Delta}$ instead of the leader fraction $L_{\Delta}$ gives similar order-of-magnitude variations with PWV for large and small counter separation.

The values of $L_{\Delta>5}^{\mathrm{xe}}$ and $L_{\Delta>5}^{\mathrm{xm}}$, already corrected for atmospheric pressure and humidity effects, are presented in Figure 3 as follower fractions $1-L_{\Delta}$ relative to the average of this period. The apparent time fluctuations of $\sim 20 \%$ or larger can only be attributed to current uncertainties of the method. No clear time dependence was found for this time interval.

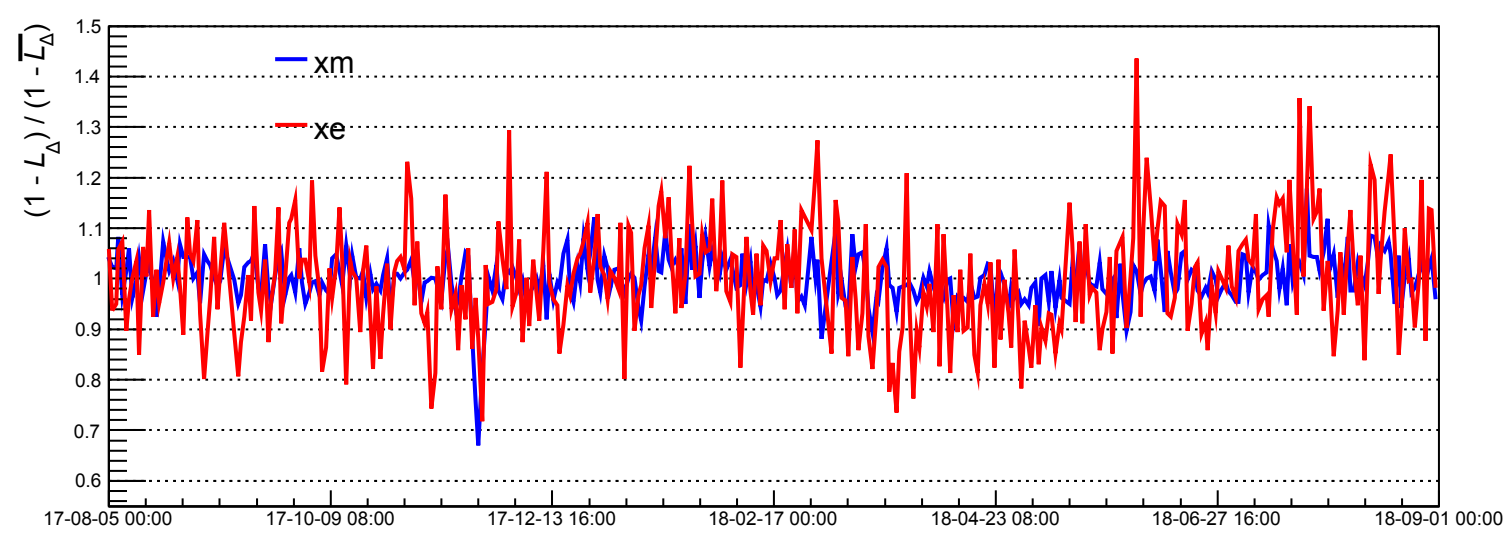

Figure 3: Relative variations of the follower fractions $1-L_{\Delta>5}^{\mathrm{xe}}$ and $1-L_{\Delta>5}^{\mathrm{xm}}$ for the time interval studied.

\section{Conclusions}

We present results from a new data-acquisition electronics system that measures cross-counter time delay histograms at the Princess Sirindhorn Neutron Monitor at the summit of Doi Inthanon, 
Thailand. For these, we have extracted cross-counter leader fractions, which are related to crosscounter neutron multiplicity. An analysis of the cross-counter leader fraction as a function of counter separation distance reveals a nearly constant cross-counter multiplicity at large counter separation, not consistent with 1 . We attribute this to multiple atmospheric secondary particles from the same primary cosmic ray. We have measured the effects of atmospheric pressure and water vapor on the cross-counter leader fraction, and corrected for these effects during August, 2017-August, 2018. After correction, no clear time dependence was found for this time period.

In future work, we will analyze Monte Carlo simulations of atmospheric particle showers to estimate the expected time delay distributions of secondary neutrons and justify that distant crosscounter multiplicity is indeed due to multiple secondaries. We will also report on data taken for individual events for which 15 or more counters present simultaneous detections (within $1 \mathrm{~ms}$ ) and explore their possible origin associated to same-primary atmospheric showers.

\section{Acknowledgments}

Partially supported by the Thailand Research Fund award RTA5980003, TRG5880173, and US National Science Foundation awards PLR-1245939 and PLR-1341562 and their predecessors.

\section{References}

[1] C. J. Hatton and H. Carmichael, Experimental investigation of the NM-64 neutron monitor, CaJPh 42 , 2443 (1964).

[2] A. Sáiz, W. Mitthumsiri, D. Ruffolo, P. Evenson and T. Nutaro, Measurement of cross-counter leader fractions in an 18NM64: Detecting single and multiple atmospheric secondaries, in proceedings of 35th International Cosmic Ray Conference, POS ( ICRC2017) 047 (2017).

[3] D. Ruffolo, A. Sáiz, P.-S. Mangeard, N. Kamyan, P. Muangha, T. Nutaro, S. Sumran, C. Chaiwattana, N. Gasiprong, C. Channok, C. Wuttiya, M. Rujiwarodom, P. Tooprakai, B. Asavapibhop, J. W. Bieber, J. Clem, P. Evenson and K. Munakata, Monitoring short-term cosmic-ray spectral variations using neutron monitor time-delay measurements, ApJ 817, 38 (2016).

[4] P.-S. Mangeard, D. Ruffolo, A. Sáiz, W. Nuntiyakul, J. W. Bieber, J. Clem, P. Evenson, R. Pyle, M. L. Duldig and J. E. Humble, Dependence of the neutron monitor count rate and time delay distribution on the rigidity spectrum of primary cosmic rays, JGRA 121, 11620 (2016).

[5] C. Banglieng, D. Ruffolo, A. Sáiz, P. Evenson and T. Nutaro, Tracking cosmic-ray spectral variations with neutron monitor time-delay measurements at high cutoff rigidity during 2007-2017, in proceedings of 35th International Cosmic Ray Conference, POS ( ICRC2017) 025 (2017).

[6] C. Banglieng, H. Janthaloet, D. Ruffolo, A. Sáiz, W. Mitthumsiri, P. Evenson, T. Nutaro, R. Pyle, S. Seunarine and J. Madsen, Neutron monitor time-delay measurements to track cosmic ray spectral variation due to solar modulation at high and low cutoff rigidity, these proceedings. 